\title{
Size- and Leaf age-dependent Effects on the Photosynthetic Physiological Responses of Artemisia ordosica to Drought Stress
}

\author{
chunyuan wang ${ }^{1}$, Minghan $\mathrm{Yu}^{1}$, Guodong Ding ${ }^{1}$, Guanglei Gao ${ }^{1}$, Linlin Zhang ${ }^{1}$, Yingying \\ $\mathrm{He}^{1}$, and Wei Liu ${ }^{1}$
}

${ }^{1}$ Affiliation not available

September 21, 2020

\begin{abstract}
Drought is one of the most significant natural disasters in the arid and semi-arid areas of China. The growth stages of populations or plant organs often differ in how they respond to drought and other adversities. At present, little is known about size- and leaf age-dependent differences in the mechanisms of shrubs-related drought resistance in China's deserts. We studied Artemisia ordosica Krasch to evaluate its photosynthesis responses to drought stress. A field experiment conducted in Mu Us Desert, Ningxia, China. Rainfall was manipulated by installing outdoor shelters, with four rainfall treatments applied to 12 plots (each 5 ? $5 \mathrm{~m}$ ). There were four precipitation levels CK (ambient), $-30 \%,-50 \%,-70 \%$, each with three replications. Taking individual crown size as the dividing basis, the responses of the plants' photosynthetic systems to drought were measured at different growth stages, i.e. large-sized ( $1 \mathrm{~m} 2)$, medium-sized (0.25-1 m2), small-sized (¡0.25 m2). In the meanwhile, leaves were divided into mature leaves and new ones for separately measurement. Our results showed that (1) under drought stress, the transfer efficiency of light energy captured by antenna pigments to the PSII reaction center decreased, and the heat dissipation capacity increased simultaneously. To resist the photosynthetic system damage caused by drought, A. ordosica enhanced free radical scavenging by activating the antioxidant enzyme system. (2) The threshold for a reduction in rainfall was $70 \%$; beyond this value, the adaptive regulation of the photosynthetic system in A. ordosica failed. (3) The growth stages and leaves age led to differences in the photosynthetic system reaction to drought. Small A. ordosica plants could not withstand severe drought stress (70\% rainfall reduction), whereas large A. ordosica individuals could absorb deep soil water to ensure their survival. Under mild drought stress, tender (younger) leaves had a greater ability to resist drought than older leaves, whereas the latter were more resistant to drought under severe stress.
\end{abstract}

\section{Hosted file}

Size- and Leaf age-dependent Effects on the Photosynthetic Physiological Responses of Artemisia ordosic available at https://authorea.com/users/360054/articles/482328-size-and-leaf-age-dependenteffects-on-the-photosynthetic-physiological-responses-of-artemisia-ordosica-to-droughtstress 